Suzanne Conradie MB CHB M MED (ANESTH) FCA, André Coetzee MB CHB MMED(ANESTH) FFA(SA) PHD FFARCS MD PhD, Johan Coetzee BSC MB CHB MMED(ANESTH) PhD

\section{Anesthetic modulation of myocardial ischemia and reperfusion injury in pigs: comparison between halothane and sevoflurane}

Purpose: Halothane offers protection against the reperfusion injury of the myocardium. This study compared sevoflurane with halothane in its potential to modulate the effects of acute severe ischemia and reperfusion on the myocardium.

Methods: Experiments were conducted on 25 pigs. Anesthesia consisted of thiopental, vecuronium and fentanyl. The lungs were mechanically ventilated with oxygen and nitrogen. Animals were randomly allocated to receive either I MAC halothane or sevoflurane. A control group received fentanyl and pentobarbital. Regional myocardial function was measured with sonomicrometers. The left anterior descending coronary artery was occluded for 15 min followed by 60 min reperfusion.

Results: Neither halothane nor sevoflurane protected the heart against the effects of acute and severe regional myocardial ischemia. During reperfusion, $89 \%$ of the animals receiving sevoflurane suffered from ventricular fibrillation compared with $30 \%$ in the halothane group $(P<0.005)$. Five minutes into the reperfusion period the animals subjected to halothane anesthesia demonstrated an $88 \%$ recovery in regional myocardial systolic function while in the sevoflurane group the recovery was $40 \%$ of pre-ischemic control $(P<0.05)$.

Conclusion: Halothane is associated with less reperfusion arrhythmias and, in addition, recovery of regional myocardial function during reperfusion was more rapid in the presence of halothane than with sevoflurane.

Objectif : L'halothane offre une protection contre les lésions reliées à la reperfusion du myocarde. La présente étude a comparé le sévoflurane et l'halothane quant au pouvoir de moduler les effets de l'ischémie aiguë sévère et de la reperfusion du myocarde.

Méthode : Les expériences ont été menées avec 25 porcs. On a utilisé du thiopental, du vécuronium et du fentanyl pour l'anesthésie. La ventilation mécanique des poumons s'est faite avec de l'oxygène et de l'azote. Les animaux, répartis de façon aléatoire, ont reçu I CAM d'halothane ou de sévoflurane. Un groupe témoin a reçu du fentanyl et du pentobarbital. La fonction régionale du myocarde a été mesurée à l'aide de sonomicromètres. L'occlusion de 15 min de l'artère interventriculaire antérieure a été suivie de 60 min de reperfusion.

Résultats : Ni l'halothane ni le sévoflurane n'ont réussi à protéger le coeur des effets d'une ischémie régionale aiguë et sévère du myocarde. Pendant la reperfusion, $89 \%$ des animaux qui avaient reçu du sévoflurane et 30 $\%$ de ceux qui avaient reçu de l'halothane ont souffert de fibrillation ventriculaire $(P<0,005)$. En cinq minutes de reperfusion, les animaux soumis à l'anesthésie à base d'halothane ont présenté un taux de récupération de la fonction systolique régionale du myocarde de $88 \%$ tandis que ceux qui avaient reçu du sévoflurane n'ont recouvré qu'à $40 \%$ l'état préischémique enregistré $(P<0,05)$.

Conclusion : Comparé au sévoflurane, l'halothane est associé à moins d'arythmie de reperfusion et à une récupération plus rapide de la fonction régionale du myocarde pendant la reperfusion.

From the Department of Anesthesiology, University of Stellenbosch Medical School, P.O. Box 19063, Tygerberg, 7505 South Africa. Address for correspondence: André Coetzee MD PhD, P.O. Box 19063, Tygerberg 7505, South Africa. Phone: 27-21-938-9226;

Fax: 27-21-938-9144; E-mail: arc1@maties.sun.ac.za

Study supported by Abbott (SA) and André Coetzee is supported by the MRC and Harry Crossley Foundation. Accepted for publication October 11, 1998 
I T has been demonstrated that halothane, enflurane and isoflurane improve systolic function during reperfusion of the isolated rat heart. ${ }^{1,2}$ Similar results were reported in a chronically instrumented dog model $^{3}$ and data from acute experiments confirmed that halothane afforded protection to the reperfused heart (less stunning) after $15 \mathrm{~min}$ total ischemia. In addition, halothane was effective in limiting reperfusion arrhythmias. ${ }^{4}$

Reperfusion injury of the heart is manifested by reversible myocardial dysfunction (stunning), arrhythmias and accelerated cell death. ${ }^{5}$ Free oxygen radical formation during reperfusion is an important etiological factor in reperfusion injury ${ }^{6}$ and drugs which counteract these radicals, attenuate myocardial stunning. ${ }^{7-9}$ Intracellular calcium overload is another feature of the stunned myocardium ${ }^{10,11}$ and it is generally accepted to be the end result of the effect of free oxygen radicals on myocytes. ${ }^{5}$ However, some authors speculate that this could represent a separate pathophysiological process. ${ }^{12}$

Based on the evidence that calcium overload is important in the reperfusion injury, and on the published effects of halothane on calcium flux across cellular and subcellular membranes, ${ }^{13-19}$ we initially studied the effects of halothane on the ischemic and reperfused heart in an open chest pig model. ${ }^{4}$ To date, similar studies have not been done for the newer inhalational agents sevo- and desflurane but experimental data suggest that sevoflurane depresses myocardial contractility because of its blocking effect on transmembrane calcium flux ${ }^{20,21}$ It is, therefore, likely that sevoflurane may have similar protective effects as were demonstrated for halothane. ${ }^{4}$

In this study, we compared sevoflurane with halothane with special reference to their ability to modify the effects of acute severe myocardial ischemia and reperfusion on regional myocardial function.

\section{Materials and methods}

This study was approved by the Ethics Committee of the Medical School of the University of Stellenbosch. Animals are kept in the animal facility of the Faculty and care conforms to national and University guidelines.

Pigs of both sexes $(21-25 \mathrm{~kg})$ were premedicated with $3 \mathrm{mg} \cdot \mathrm{kg}^{-1} \mathrm{ketamine} i \mathrm{~m}$. Intravenous access was established via an ear vein and the animals received 25 $\mathrm{ml} \cdot \mathrm{kg}^{-1} \cdot \mathrm{hr}^{-1}$ normal saline for the duration of the procedure. Thiopental $\left(2 \mathrm{mg} \cdot \mathrm{kg}^{-1}\right)$ was given iv and anesthesia was further maintained with either halothane (Rhone-Poulenc Rorer, South Africa) or sevollurane (Ultane, Abbott Laboratories, Ireland) in oxygen using a circle system with a carbon dioxide $\left(\mathrm{CO}_{2}\right)$ absorber in the circuit and a fresh gas flow of $6 \mathrm{~L} \cdot \mathrm{min}^{-1}$. When the animal was unresponsive, a tracheostomy was performed, the trachea intubated and the lungs ventilated (Spiromat, Drager, Germany) with nitrogen and oxygen (one L. min $^{-1}$ each). The initial tidal volume was 10 $\mathrm{ml} \cdot \mathrm{kg}^{-1}$ and the rate 15 breaths per min. The tidal volume was adjusted to maintain the end-expired $\mathrm{CO}_{2}$ concentration between $4.5-5.0 \%$ (Cardiocap, Datex, Finland). Muscle relaxation was obtained using vecuronium $0.2 \mathrm{mg} \cdot \mathrm{kg}^{-1}$ initially and thereafter 0.3 $\mathrm{mg} \cdot \mathrm{kg}^{-1} \cdot \mathrm{hr}^{-1}$ as a constant infusion). Body temperature was maintained between 36.5 and $37.5^{\circ} \mathrm{C}$ with the aid of an undertable heating device.

Anesthesia was maintained with an initial bolus of fentanyl $\left(20 \mu \mathrm{g} \cdot \mathrm{kg}^{-1}\right)$ followed by a constant infusion of $10 \mu \mathrm{g} \cdot \mathrm{kg}^{-1} \cdot \mathrm{hr}^{-1}$. Halothane or sevoflurane was administered from calibrated vaporisers (Fluotec, Cyprane, UK; Penlon, Penlon Ltd., UK). The anesthetic agent concentrations were monitored (Ohmeda 5330 Agent Monitor, Ohmeda, USA) and the vaporiser was continuously adjusted to maintain an endexpired agent concentration of $1 \mathrm{MAC}$ (halothane $0.7 \%$, sevoflurane $1.7 \%$ ).

In the control group (five animals) anesthesia consisted of the fentanyl infusion (as for the halothane and sevoflurane groups) and an intravenous bolus of pentobarbital ( $\left.8 \mathrm{mg} \cdot \mathrm{kg}^{-1}\right)$ followed by a constant infusion of $3 \mathrm{mg} \cdot \mathrm{kg}^{-1} \cdot \mathrm{hr}^{-1}$ pentobarbital.

The neck was dissected and a short 16-gauge catheter inserted into the carotid artery and advanced to within $3 \mathrm{~cm}$ of the aortic valve. This was connected to a transducer (Medex, Medex Med Inc. UK) and used to display systolic and diastolic arterial pressures (SAP, DAP) (Cardiocap, Datex, Finland). Lead I of the ECG was monitored continuously.

A pulmonary artery catheter (7 F Arrow, Arrow, USA) was placed in the proximal pulmonary artery via the internal jugular vein. The cardiac output (CO) was determined by thermodilution $(5 \mathrm{ml}$ dextrose $5 \%$ injected by hand within four seconds). Three injections were spaced throughout the respiratory cycle and the CO computer (Edwards REF, Baxter, USA) calculated the average value which was used in subsequent calculations.

The right femoral area was dissected and an occlusion catheter (Fogarty, Baxter, USA) was inserted into the femoral artery and advanced into the descending thoracic aorta. This balloon was used to raise left ventricular (LV) afterload for the determination of endsystolic LV elastance (Ees) as described below.

A sternotomy was performed and the pericardium opened. A short stiff 16-gauge cannula, connected to a pressure transducer (Medex, Medex Ltd., UK) was 
TABLE I General hemodynamic in the three experimental groups.

\begin{tabular}{|c|c|c|c|c|c|}
\hline & Control & Ischemia & Reperf +5 & Reperf +30 & Reperf +6 \\
\hline \multicolumn{6}{|c|}{$\operatorname{CONTROL}(n=5)$} \\
\hline $\mathrm{HR}$ & 120 & 109 & 119 & 107 & 107 \\
\hline$\left(b \cdot \min ^{-1}\right)$ & $(113-124)$ & $(105-121)$ & $(115-121)$ & $(105-117)$ & $(101-115)$ \\
\hline MAP & 77 & 81 & 74 & & 80 \\
\hline (mmHg) & $(69-87)$ & $(64-90)$ & $(58-86)$ & $(69-72)$ & $(63-99)$ \\
\hline LVEDP & 5.2 & 4.4 & 9.4 & 6.3 & 6.4 \\
\hline$(\mathrm{mmHg})$ & $(2.7-9.4)$ & $(11.2-17.9)$ & $(7.3-13)$ & $(3.6-9.3)$ & $(6-7.9)$ \\
\hline $\mathrm{CO}$ & 3.4 & 3.6 & 3.9 & & 3.4 \\
\hline $\left.1 \cdot \min ^{-1}\right)$ & $(2.9-3.9)$ & $(3.9-3.8)$ & $(3.3-4.1)$ & $(2.3-3.3)$ & $(3.0-4.2)$ \\
\hline \multicolumn{6}{|c|}{ HALOTHANE $(\mathrm{n}=10)$} \\
\hline HR & 93 & 89 & 89 & 91 & 89 \\
\hline$\left(\mathrm{b} \cdot \mathrm{min}^{-1}\right)$ & $(82-100)$ & $(81-105)$ & $(80-89)$ & $(81-110)$ & $(85-113)$ \\
\hline MAP & 72 & 63 & 70 & 79 & 74 \\
\hline$(\mathrm{mmHg})$ & $(64-77)$ & $(56-69)$ & $(66-75)$ & $(63-71)$ & $(69-79)$ \\
\hline LVEDP & 11.1 & 12.6 & 9.9 & 9.3 & 10.6 \\
\hline$(\mathrm{mmHg})$ & $(7.8-13)$ & $(9.3-17.0)$ & $(5.9-11.6)$ & $(6.1-13.9)$ & $(7.1-15.4)$ \\
\hline $\mathrm{CO}$ & 2.5 & $2.1^{*}$ & $2.7 \dagger$ & 2.7 & 2.1 \\
\hline$\left(1 \cdot \min ^{-1}\right)$ & $(2.4-2.9)$ & $(1.8-2.5)$ & $(2.1-3.2)$ & $(2.1-2.9)$ & $(1.8-3.0)$ \\
\hline \multicolumn{6}{|c|}{ SEVOFLURANE $(n=9)$} \\
\hline HR & 88 & 97 & 103 & 94 & 88 \\
\hline (b-min'-1) & $(82-100)$ & $(82-104)$ & $(88-107)$ & $(81-110)$ & $(77-103)$ \\
\hline MAP & 82 & 76 & 77 & 71 & 73 \\
\hline$(\mathrm{mmHg})$ & $(72-98)$ & $(66.79)$ & $(70-81)$ & $(65-80)$ & $(63-81)$ \\
\hline LVEDP & 11.4 & 12.4 & 9.9 & 9.1 & 9.8 \\
\hline$(\mathrm{mmHg})$ & $(6.2-13.7)$ & $(8.5-15.2)$ & $(8.1-14.4)$ & $(5.6-11.4)$ & $(4.1-12.7)$ \\
\hline $\mathrm{CO}$ & 2.5 & 2.4 & 2.6 & 2.2 & $2.3^{\mathrm{s}}$ \\
\hline$\left(1 \cdot \min ^{-1}\right)$ & $(2.3-3.1)$ & $(2.1-2.6)$ & $(2.2-3.0)$ & $(2.0-2.7)$ & $(1.8-2.5)$ \\
\hline
\end{tabular}

Data are presented as median $\left(25^{\text {th }} \cdot 75^{\text {th }}\right.$ percentiles $)$

Reperf $=$ reperfusion at 5, 30 and $60 \mathrm{~min}$.

$\mathrm{HR}$ = heart rate; $\mathrm{MAP}=$ mean arterial pressure; $\mathrm{LVEDP}=$ left ventricular end-diastolic pressure; $C O=$ cardiac output.

* = Differs from control

$\dagger$ - Differs from ischemia

inserted into the $L V$ cavity via a stab incision in the $L V$ apex. The catheter was sutured to the myocardium and was used to determine $L V$ peak, $L V$ end-diastolic (LVEDP) and end-systolic pressure (Pes). Two pairs of sonomicrometer crystals were inserted into the subendocardium: one pair in the region supplied by the left anterior descending coronary artery (LAD artery) and the other pair in the region supplied by the circumflex coronary artery (LX artery). Signals were transduced (Schuessler and Ass, USA) and used to determine regional systolic shortening (dL). Shortening which occurred after the aortic valve closed (taken at the dicrotic notch of the aortic pressure recording) was termed post-systolic shortening (PSS) and was expressed as a percentage of the total segmental shortening. Signals were combined with $L V$ pressure to give real time pressure-length (P-L) loops for the areas into which the crystals had been inserted. By raising the $\mathrm{LV}$ afterload using the occlusion catheter, a series of changing P-L loops could be generated from which various regional cardiac parameters were calculated as described below.

The LAD coronary artery was dissected from the epicardium distal to the first diagonal branch and a soft silastic loop placed around the artery. This was used to cause total occlusion of the LAD artery for $15 \mathrm{~min}$ as part of the experimental protocol. Heparin, $1 \mathrm{mg} \cdot \mathrm{kg}^{-1}$, was administered $i v$ after surgery was completed.

\section{Protocol}

Animals were allocated to either the halothane or the sevoflurane group by means of a coin toss. In addition, five control experiments were performed. The control group did not receive inhalational anesthesia and was not part of the formal randomisation.

After $15 \mathrm{~min}$ stabilisation, control recordings were made. The LAD coronary artery then was occluded for 
TABLE II Regional myocardial function in the three experimental groups

\begin{tabular}{|c|c|c|c|c|c|c|}
\hline & Control & Ischemia & $R e p e r f+5$ & Reperf +30 & Reperf +60 & $P$ \\
\hline \multicolumn{7}{|c|}{ CONTROL $(n=5)$} \\
\hline $\begin{array}{l}\text { LAD dL } \\
(\mathrm{mm})\end{array}$ & $\begin{array}{l}1.2 \\
(0.6-1.4)\end{array}$ & $\begin{array}{l}0.06^{*} \\
(-0.04-0.21)\end{array}$ & $\begin{array}{l}0.5^{*} \\
(0.04-0.06)\end{array}$ & $\begin{array}{l}0.7 \\
(0.4-0.9)\end{array}$ & $\begin{array}{l}0.8 \\
(0.6-1.0)\end{array}$ & 0.001 \\
\hline $\begin{array}{l}\mathrm{LAD} d \mathrm{dL} \\
(\%)\end{array}$ & $\begin{array}{l}15.6 \\
(9.9-23.1)\end{array}$ & $\begin{array}{l}0.9^{*} \\
(-0.5-3.3)\end{array}$ & $\begin{array}{l}8.4^{*} \\
(5.8-9.6)\end{array}$ & $\begin{array}{l}11.1 \\
(5.4-12.6)\end{array}$ & $\begin{array}{l}12.1 \\
(8.0-13.7)\end{array}$ & 0.001 \\
\hline $\begin{array}{l}\text { LAD PSS } \\
(\%)\end{array}$ & 0 & $\begin{array}{l}85^{*} \\
(77.5-112.5)\end{array}$ & $\begin{array}{l}19.4 \\
(7.6-32.7)\end{array}$ & $\begin{array}{l}7.5 \\
(5.5-30.5)\end{array}$ & $\begin{array}{l}3.4 \dagger \\
(0-9.6)\end{array}$ & 0.001 \\
\hline $\begin{array}{l}\text { LAD RSW } \\
\left(\mathrm{mmHg} \cdot \mathrm{mm}^{-1}\right)\end{array}$ & $\begin{array}{l}76 \\
(34-103)\end{array}$ & $\begin{array}{l}5^{*} \\
(-3-11)\end{array}$ & $\begin{array}{l}33 \\
(30.47)\end{array}$ & $\begin{array}{l}46 \\
(26-78)\end{array}$ & $\begin{array}{l}58 \dagger \\
(48-84)\end{array}$ & 0.002 \\
\hline $\begin{array}{l}\text { LAD Ees } \\
\left(\mathrm{mmHg} \cdot \mathrm{mm}^{-1}\right)\end{array}$ & $\begin{array}{l}71 \\
(59-153)\end{array}$ & $\begin{array}{l}108 \\
(71-135)\end{array}$ & $\begin{array}{l}83 \\
(63-123)\end{array}$ & $\begin{array}{l}44 \\
(35-76)\end{array}$ & $\begin{array}{l}62 \\
(42-79)\end{array}$ & 0.29 \\
\hline $\begin{array}{l}\text { LAD Lo } \\
(\mathrm{mm})\end{array}$ & $\begin{array}{l}3.3 \\
(2.7-4.5)\end{array}$ & $\begin{array}{l}5.5 \\
(4.3-6.2)\end{array}$ & $\begin{array}{l}5.3 \\
(5.1-5.7)\end{array}$ & $\begin{array}{l}3.9 \\
(3.6-4.9)\end{array}$ & $\begin{array}{l}4.7 \\
(3.5-4.9)\end{array}$ & 0.29 \\
\hline \multicolumn{7}{|c|}{ HALOTHANE $(n=10)$} \\
\hline $\begin{array}{l}\mathrm{LAD} d \mathrm{dL} \\
(\mathrm{mm})\end{array}$ & $\begin{array}{l}0.5 \\
(0.34-0.71)\end{array}$ & $\begin{array}{l}0.08^{*} \\
(0.03-0.24)\end{array}$ & $\begin{array}{l}0.38 \dagger \\
(0.30-0.47)\end{array}$ & $\begin{array}{l}0.42 \dagger \\
(0.33-0.69)\end{array}$ & $\begin{array}{l}0.40 \dagger \\
(0.29-0.77)\end{array}$ & 0.018 \\
\hline $\begin{array}{l}\text { LAD dL } \\
\text { (\%) }\end{array}$ & $\begin{array}{l}9.2 \\
(7.4-11.6)\end{array}$ & $\begin{array}{l}1.5^{*} \\
(0.6-4.2)\end{array}$ & $\begin{array}{l}7.3 \dagger \\
(6.4-8.2)\end{array}$ & $\begin{array}{l}8.1 \dagger \\
(6.0-10.9)\end{array}$ & $\begin{array}{l}7.3 \dagger \\
(5.9-13.1)\end{array}$ & 0.007 \\
\hline $\begin{array}{l}\text { LAD PSS } \\
(\%)\end{array}$ & 0 & $\begin{array}{l}51.9^{*} \\
(25.0-61.6)\end{array}$ & $\begin{array}{l}8.6 \\
(0.0-14.7)\end{array}$ & $\begin{array}{l}3.5 \dagger \\
(0-13.5)\end{array}$ & $\begin{array}{l}6.5 \\
(0-17.8)\end{array}$ & 0.001 \\
\hline $\begin{array}{l}\mathrm{LAD} R S W \\
\left(\mathrm{mmHg} \cdot \mathrm{mm}^{-1}\right)\end{array}$ & $\begin{array}{l}30 \\
(24-43)\end{array}$ & $\begin{array}{l}4^{*} \\
(2-21)\end{array}$ & $\begin{array}{l}24 \\
(19-28)\end{array}$ & $\begin{array}{l}24 \dagger \\
(17-43)\end{array}$ & $\begin{array}{l}27 \dagger \\
(19-52)\end{array}$ & 0.012 \\
\hline $\begin{array}{l}\text { LAD Ees } \\
\left(\mathrm{mmHg} \cdot \mathrm{mm}^{-1}\right)\end{array}$ & $\begin{array}{l}160 \\
(116-219)\end{array}$ & $\begin{array}{l}303 \\
(219-456)\end{array}$ & $\begin{array}{l}121 \\
(100-226)\end{array}$ & $\begin{array}{l}98 \dagger \\
(68-115)\end{array}$ & $\begin{array}{l}97 \dagger \\
(61-171)\end{array}$ & 0.001 \\
\hline $\begin{array}{l}\text { LAD Lo } \\
(\mathrm{mm})\end{array}$ & $\begin{array}{l}4.1 \\
(3.9-4.8)\end{array}$ & $\begin{array}{l}5.1^{*} \\
(4.8-5.9)\end{array}$ & $\begin{array}{l}4.4 \dagger \\
(4.1-4.8)\end{array}$ & $\begin{array}{l}4.0 \dagger \\
(3.8-4.7)\end{array}$ & $\begin{array}{l}4.2 \dagger \\
(4.1-4.5)\end{array}$ & 0.001 \\
\hline \multicolumn{7}{|c|}{ SEVOFLURANE $(n=9)$} \\
\hline $\begin{array}{l}\mathrm{LAD} d \mathrm{dL} \\
(\mathrm{mm})\end{array}$ & $\begin{array}{l}0.54 \\
(0.52-0.87)\end{array}$ & $\begin{array}{l}0.10^{\star} \\
(0.09-0.18)\end{array}$ & $\begin{array}{l}0.28^{*} \dagger \\
(0.19-0.42)\end{array}$ & $\begin{array}{l}0.29^{*} t \\
(0.19-0.52)\end{array}$ & $\begin{array}{l}0.49^{\star} \dagger \\
(0.29-0.56)\end{array}$ & 0.001 \\
\hline $\begin{array}{l}\text { LAD dL } \\
\text { (\%) }\end{array}$ & $\begin{array}{l}10.9 \\
(9.5-14.3)\end{array}$ & $\begin{array}{l}1.8^{*} \\
(1.8-2.7)\end{array}$ & $\begin{array}{l}4.9^{\star} \\
(4.0-6.6)\end{array}$ & $\begin{array}{l}5.1^{*} \\
(3.1-9.3)\end{array}$ & $\begin{array}{l}8.8^{\star} t \\
(4.8-9.7)\end{array}$ & 0.001 \\
\hline $\begin{array}{l}\text { LAD PSS } \\
(\%)\end{array}$ & 0 & $\begin{array}{l}44.4^{*} \\
(21.4-60.6)\end{array}$ & $\begin{array}{l}2.1 \\
(0-21.5)\end{array}$ & $\begin{array}{l}7.6 \\
(0-30.0)\end{array}$ & $\begin{array}{l}0 \dagger \\
(0-1.7)\end{array}$ & 0.001 \\
\hline $\begin{array}{l}\text { LAD RSW } \\
\left(\mathrm{mmHg} \cdot \mathrm{mm}^{-1}\right)\end{array}$ & $\begin{array}{l}52 \\
(37-77)\end{array}$ & $\begin{array}{l}8^{\star} \\
(7-14)\end{array}$ & $\begin{array}{l}19^{*} \\
(16-30)\end{array}$ & $\begin{array}{l}22^{*} \\
(11-36)\end{array}$ & $\begin{array}{l}37 \dagger \\
(22-38)\end{array}$ & 0.001 \\
\hline $\begin{array}{l}\text { LAD Ees } \\
\left(\mathrm{mmHg} \cdot \mathrm{mm}^{-1}\right)\end{array}$ & $\begin{array}{l}190 \\
(134-282)\end{array}$ & $\begin{array}{l}252 \\
(156-1342)\end{array}$ & $\begin{array}{l}190 \\
(160-309)\end{array}$ & $\begin{array}{l}141 \\
(99-251)\end{array}$ & $\begin{array}{l}108 \dagger \\
(85-169)\end{array}$ & 0.004 \\
\hline $\begin{array}{l}\text { LAD Lo } \\
(\mathrm{mm})\end{array}$ & $\begin{array}{l}4.5 \\
(3.5-8.4)\end{array}$ & $\begin{array}{l}5.0 \\
(4.4-9.4)\end{array}$ & $\begin{array}{l}5.4 \\
(4.4-9.7)\end{array}$ & $\begin{array}{l}5.2 \\
(4.4-9.1)\end{array}$ & $\begin{array}{l}5.0 \\
(4.2-9.5)\end{array}$ & 0.41 \\
\hline
\end{tabular}

Data are presented as median ( $25^{\text {th }}$ to $75^{\text {th }}$ percentiles).

Reperf $=$ reperfusion at 5,30 and $60 \mathrm{~min}$.

$\mathrm{LAD}=$ left arterior descending coronary artery.

$\mathrm{dL}=$ regional systolic shortening; $\mathrm{dL} \%=$ regional systolic shortening normalized for max. segment length; PSS = post systolic shortening; $\mathrm{RSW}=$ regional stroke work; Ees $=$ end-systolic elastance; $L o=$ extrapolated segment length when $L V$ pressure $=0$.

$P=$ repeated measures analysis of variance

* = Differs from control

$t=$ Differs from ischemia

$15 \mathrm{~min}$. At $10 \mathrm{~min}$ after occlusion, recordings were made (ischemia). The occlusion was released at $15 \mathrm{~min}$ and, five minutes after reperfusion was established, recordings were repeated (reperfusion $+5 \mathrm{~min}$.). Thereafter recordings were performed at 30 and 60 $\mathrm{min}$ after establishing reperfusion (reperfusion $+30 \mathrm{~min}$ and reperfusion $+60 \mathrm{~min}$ ).
If more than five ventricular ectopic beats per minute occurred or if they were multifocal in origin, 1 $\mathrm{mg} \cdot \mathrm{kg}^{-1}$ iv lidocaine was administered. This was repeated if necessary.

Ventricular tachycardia and ventricular fibrillation were treated with $1 \mathrm{mg} \cdot \mathrm{kg}^{-1}$ lidocaine iv followed by direct defibrillation of the heart with $5,5,10,10$ and 
TABLE III Treatment of reperfusion arrhythmias

\begin{tabular}{lllll}
\hline & Halothane & Sevoflurane & Control & $P$ \\
Mass & group $(\mathrm{n}=10)$ & group $(\mathrm{n}=9)$ & group $(\mathrm{n}=5)$ & \\
$(\mathrm{kg})$ & 22.5 & 24 & 24 & 0.48 \\
Lidocaine & $(20-24)$ & $(22.7-25)$ & $(20.7-24)$ & \\
$(\mathrm{mg})$ & $0^{*}$ & 30 & 40 & 0.001 \\
Joules & $(0-20)$ & $(25.7-40)$ & $(30.5-49)$ & \\
& $0 \dagger$ & 30 & 50 & 0.001 \\
Number of animals requiring defibrillation & $(0-15)$ & $(15.5-40)$ & $(43.7-62.5)$ & $0.005 \ddagger$ \\
\hline
\end{tabular}

Results are given as median $\left(25^{\text {th }}\right.$ and $75^{\text {th }}$ percentile).

* Significantly different from sevoflurane and control groups.

$\dagger$ Significantly different from control group but not from sevoflurane group.

$\ddagger$ Chi-squared $=10.55$ with 2 degrees of freedom.

TABLE IV Intergroup comparison

\begin{tabular}{|c|c|c|c|c|c|}
\hline Variable & Group & Ischemia & Reperf +5 & Reperf +30 & Reperf +60 \\
\hline \multirow[t]{3}{*}{$\begin{array}{l}\text { LAD dL } \\
(\%)\end{array}$} & $\mathrm{H}$ & $\begin{array}{l}16 \\
(7-38)\end{array}$ & $\begin{array}{l}88 \\
(72-106)\end{array}$ & $\begin{array}{l}92 \\
(71-126)\end{array}$ & $\begin{array}{l}92 \\
(67-126)\end{array}$ \\
\hline & $S$ & $\begin{array}{l}19 \\
(16-23)\end{array}$ & $\begin{array}{l}40 \\
(32-62)\end{array}$ & $\begin{array}{l}56 \\
(34-71)\end{array}$ & $\begin{array}{l}65 \\
(54-74)\end{array}$ \\
\hline & $C$ & $\begin{array}{l}13 \\
(-3-21)\end{array}$ & $\begin{array}{l}55 \\
(42-62)\end{array}$ & $\begin{array}{l}64 \\
(56-74)\end{array}$ & $\begin{array}{l}80 \\
(73-88)\end{array}$ \\
\hline \multirow[t]{3}{*}{$\begin{array}{l}\text { LAD dL\% } \\
(\%)\end{array}$} & $\mathbf{H}$ & $\begin{array}{l}15 \\
(7-13)\end{array}$ & $\begin{array}{l}88^{*} \\
(68-100)\end{array}$ & $\begin{array}{l}92^{\star} \\
(71-121)\end{array}$ & $\begin{array}{l}90 \\
(65-125)\end{array}$ \\
\hline & $S$ & $\begin{array}{l}19 \\
(15-22)\end{array}$ & $\begin{array}{l}40 \\
(32-58)\end{array}$ & $\begin{array}{l}56 \\
(34-72)\end{array}$ & $\begin{array}{l}63 \\
(55-72\end{array}$ \\
\hline & $\mathrm{C}$ & $\begin{array}{l}13 \\
(-3-19)\end{array}$ & $\begin{array}{l}42 \\
(41-63)\end{array}$ & $\begin{array}{l}52 \\
(50-74)\end{array}$ & $\begin{array}{l}75 \\
(66-81)\end{array}$ \\
\hline \multirow[t]{3}{*}{$\begin{array}{l}\text { LAD RSW } \\
\text { (\%) }\end{array}$} & $\mathrm{H}$ & $\begin{array}{l}13 \\
(7-40)\end{array}$ & $\begin{array}{l}97^{\star} \\
(65-112)\end{array}$ & $\begin{array}{l}82^{\star} \\
(67-122)\end{array}$ & $\begin{array}{l}108^{\star} \\
(84-120)\end{array}$ \\
\hline & $S$ & $\begin{array}{l}17 \\
(14-22)\end{array}$ & $\begin{array}{l}36 \\
(26-61)\end{array}$ & $\begin{array}{l}36 \\
(26-68)\end{array}$ & $\begin{array}{l}54 \\
(44-69)\end{array}$ \\
\hline & $C$ & $\begin{array}{l}12 \\
(-3-21)\end{array}$ & $\begin{array}{l}70 \\
(42-73)\end{array}$ & $\begin{array}{l}71 \\
(54-94)\end{array}$ & $\begin{array}{l}107 \\
(84-127)\end{array}$ \\
\hline \multirow[t]{3}{*}{$\begin{array}{l}\text { LAD Lo } \\
\text { (\%) }\end{array}$} & $\mathrm{H}$ & $\begin{array}{l}120 \\
(110-126)\end{array}$ & $\begin{array}{l}105 \dagger \\
(98-114)\end{array}$ & $\begin{array}{l}100 \\
(96-103)\end{array}$ & $\begin{array}{l}99 \\
(82-108)\end{array}$ \\
\hline & $S$ & $\begin{array}{l}117 \\
(113-130)\end{array}$ & $\begin{array}{l}113 \\
(109-120)\end{array}$ & $\begin{array}{l}113 \\
(105-126)\end{array}$ & $\begin{array}{l}107 \\
(99-123)\end{array}$ \\
\hline & $\mathrm{C}$ & $\begin{array}{l}156 \\
(106-208)\end{array}$ & $\begin{array}{l}\text { i53 } \\
(130-198)\end{array}$ & $\begin{array}{l}\mathrm{i} 13 \\
(69-142)\end{array}$ & $\begin{array}{l}114 \\
(89-187)\end{array}$ \\
\hline \multirow[t]{3}{*}{$\begin{array}{l}\text { LAD PSS } \\
(\%)\end{array}$} & $\mathrm{H}$ & $\begin{array}{l}51 \\
(25-60)\end{array}$ & $\begin{array}{l}9 \\
(0-15)\end{array}$ & $\begin{array}{l}3 \\
(0-13)\end{array}$ & $\begin{array}{l}7 \\
(0.18)\end{array}$ \\
\hline & $S$ & $\begin{array}{l}44 \dagger \\
(21-61)\end{array}$ & $\begin{array}{l}2 \\
(0-22)\end{array}$ & $\begin{array}{l}8 \\
(0-30)\end{array}$ & $\begin{array}{l}0 \\
(0-2)\end{array}$ \\
\hline & c & $\begin{array}{l}85 \\
(77-113)\end{array}$ & $\begin{array}{l}19 \\
(8-33)\end{array}$ & $\begin{array}{l}8 \\
(6-30)\end{array}$ & $\begin{array}{l}3 \\
(0-10)\end{array}$ \\
\hline
\end{tabular}

Values are normalized for the control value and expressed as a \% of the control value. Controls are not shown as they all are $100 \%$ Values are the median and $25^{\text {th }}$ and $75^{\text {th }}$ percentiles.

$\mathrm{H}=$ halothane; $\mathrm{S}=$ sevoflurane; $\mathrm{C}=$ control; Reperf $=$ reperfusion and 5,30 and 60 minutes.

* Differs from sevoflurane group.

$\dagger$ Differs from control group. 

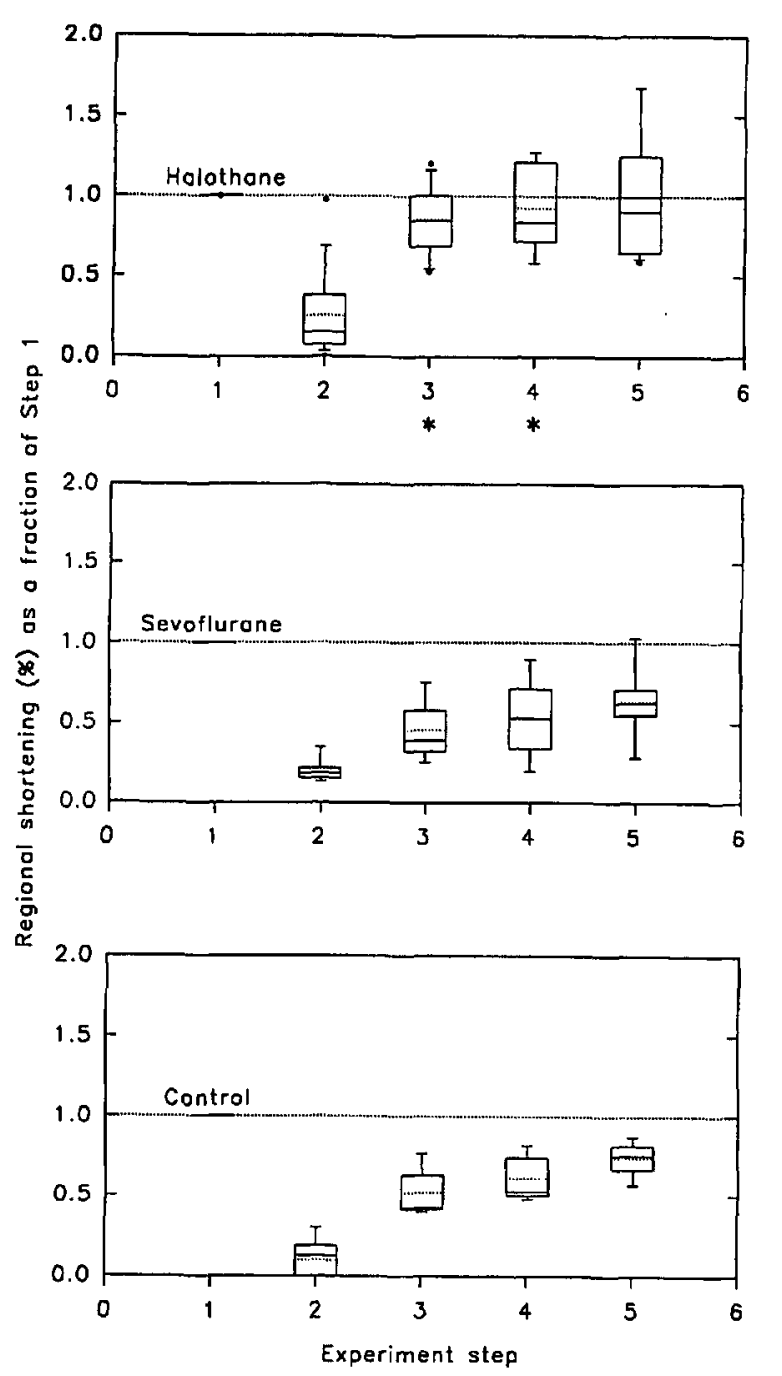

FIGURE 1 Box and Whisker plots of regional shortening of the ischemic segment in the three experimental groups.

Values are expressed as a fraction of the initial step.

The solid horizontal line within the box indicates the median value for the step, the dotted line the mean. The lower and upper edges of the box indicate the 25 and 75 th percentiles respectively. The whiskers indicate the 5 and 95 th percentiles.

* = Significantly different from the sevoflurane group.

15 joules. The total amount of lidocaine and joules required to control the ventricular arrhythmias were recorded for each animal.

At the end of the experiment the heart was arrested with $\mathrm{KCl} i v$ while the animal was maintained under deep anesthesia.

\section{Recordings and calculations}

All transduced signals (pressure, length, ECG) were digitised at a sampling rate of $100 \mathrm{~Hz}\left(\mathrm{ALAB},{ }^{*}\right.$ Central

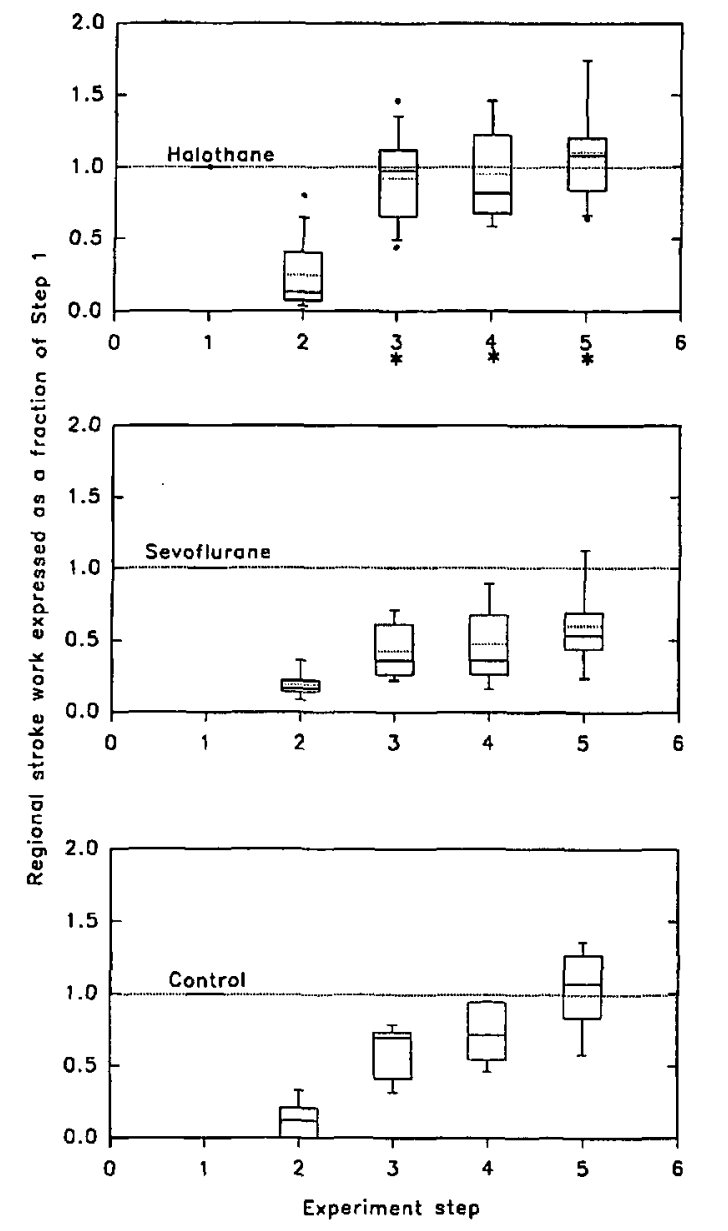

FIGURE 2 Box and whisker plot of regional stroke work in the LAD segment in the three groups.

Please see legend to Figure 1 for explanation of the box-andwhisker plots.

* = Significantly different from the sevoflurane group.

Electronic Services, University of Stellenbosch, South Africa. ALAB is a hardware-software program created by Paul Bailey (Professional Engineer) for displaying, recording and processing electronic signals obtained from monitoring and laboratory equipment). Signals were displayed graphically in real time by computer (80486Dx4 microprocessor with 4 megabytes RAM memory running at $66 \mathrm{MHZ}$ under Microsoft DOS version 6.2). At each experiment step, $30 \mathrm{sec}$ of data were recorded to computer disk at zero airway pressure. After each recording, data were redisplayed on the computer screen to evaluate the quality of the measurements. ALAB was further employed to process the recorded data off-line and to calculate the various cardiovascular parameters as described below. 
The following were recorded at each step of the protocol:

Heart rate (HR), SAP, DAP, mean arterial pressure (MAP, the area under the aortic pressure curve), LVEDP, LV end-systolic pressure (Pes), LV rate of pressure increase during isovolemic contraction $(\mathrm{dp} / \mathrm{dt})$, rate of $\mathrm{LV}$ pressure decline during isovolemic relaxation $(-\mathrm{dp} / \mathrm{dt}), \mathrm{CO}$, maximum segment length (Lmax), minimum segment length (Lmin).

The following were calculated:

End-systolic elastance (Ees): The computer defined the maximum pressure-length ratio, which occurred at end- systole, for the series of afterloaded beats. The resulting sets of end-systolic pressure and length coordinates were subjected to linear regression and the slope of the line termed Ees. The intersection of the regression line on the $x$-axis (length) was termed Lo (the extrapolated regional length at the time LV pressure is zero).

Stroke volume $(\mathrm{SV})=\mathrm{CO} / \mathrm{HR} \times 1000)(\mathrm{ml})$

Global LV stroke work (SW $=1.36$ (MAP-LVEDP) $\mathrm{x} S \mathrm{~V} / 100$ (g.m)

Regional systolic shortening $(\mathrm{dL})=\mathrm{Lmax}-\mathrm{Lmin}$ ( $\mathrm{mm}$ )

Normalised regional shortening $(\mathrm{dL} \%)=\mathrm{dL} / \mathrm{L} \max$ x $100(\%)$

Post-systolic shortening (PSS) $=$ (Segmental shortening which occurs after aortic valve closure/total segmental shortening) x $100(\%)$

Regional stroke work $(\mathrm{RSW})=$ Pes $\mathrm{x} \mathrm{dL}(\mathrm{mm}$ Hg.mm)

\section{Statistical analysis}

Statistical analyses were performed using a software package (Sigmastat for DOS 5 ,

Jandel Scientific Software, USA). Because data did not consistently conform to a normal distribution (Kolmogorov-Smirnov tests) and variances were not consistently equal (Levene median test), medians were compared using non-parametric procedures.

Within group analysis was performed using Friedman repeated measures analysis of variance (ANOVA) followed by post-hoc multiple comparisons using the Student-Newman-Keuls test. In order to conduct intergroup comparisons at each experimental step, data of each animal was expressed as a fraction of the value obtained at step 1 (the control value) and normalised values were used for Kruskall-Wallis oneway analysis of variance followed by post-hoc multiple comparisons. Enumerated data were compared by calculating $\mathrm{Chi}^{2}$ of a $2 \times 3$ contingency table. An alpha value $<0.05$ was accepted indicating a significant difference between medians.

\section{Results}

Repeated measurements, control group: (Tables $I, I I)$

Indices of global cardiac function:

There were no changes in HR, MAP, LVEDP, CO, SV, SW, $+\mathrm{dp} / \mathrm{dt}$ and $-\mathrm{dp} / \mathrm{dt}$.

Regional myocardial function:

In the ischemic segment (LAD) $\mathrm{dL}$ and $\mathrm{dL} \%$ decreased during ischemia and remained depressed five minutes after reperfusion was started. At 30 and $60 \mathrm{~min}$ reperfusion, these values improved but were not different from those of the ischemic period. The RSW decreased during ischemia and recovered slowly and by $60 \mathrm{~min}$ post-perfusion, the values were greater than those of the ischemic period were and similar to control values. There were no changes in Lmax, Ees or Lo.

There was no PSS before the onset of ischemia. During ischemia marked PSS occurred. Five minutes after reperfusion, PSS persisted in four out of the five animals and decreased progressively until it was similar to control values by $60 \mathrm{~min}$ post-perfusion when two of the five animals had zero PSS.

Repeated measurements, balothane group : (Tables I, II).

Indices of global cardiac function:

There were no changes in HR, MAP and LVEDP. During ischemia $\mathrm{CO}, \mathrm{SW},+\mathrm{dp} / \mathrm{dt}$ and $-\mathrm{dp} / \mathrm{dt}$ decreased and then recovered to values similar to control within five minutes of reperfusion.

Regional myocardial function:

In the LAD segment the following measurements decreased during ischemia and then recovered within five minutes of reperfusion, remaining at values similar to control for the rest of the duration of the experiments: dL, dL\%, RSW. Similarly, Lmax and Lo increased during ischemia and then decreased to values similar to control within five minutes.

There was no PSS present before ischemia. During ischemia, PSS increased and, five minutes after reperfusion, PSS continued in eight of the 10 animals. By 30 min post-ischemia, PSS was less than during the ischemic period and by $60 \mathrm{~min}$ four of the 10 subjects had zero PSS.

The Ees increased numerically during ischemia but did not achieve statistical significance. After reperfusion, Ees was similar to control but less than values recorded during ischemia.

There were no changes in the non-ischemic (LX) segment.

Repeated measures, sevoflurane group: (Table I, II).

Ventricular fibrillation occurred in one animal before the onset of ischemia. Data from this experiment were not used in the analysis.

Indices of global cardiac function: 
There were no changes in HR, MAP or SW. The $\mathrm{CO}$ and SV did not change during ischemia but were less than control $60 \mathrm{~min}$ after reperfusion.

Regional myocardial function:

In the LAD segment, $\mathrm{dL} \%$ and SW decreased during ischemia. These values did not improve by five and $30 \mathrm{~min}$ after reperfusion was initiated. At $60 \mathrm{~min}$ reperfusion, they had increased to values greater than those of the ischemic period but were less than those at the control stage. $\mathrm{dL}$ decreased during ischemia and increased by five minutes reperfusion although the latter remained less than those of control for the duration of the experiment. There were no changes in Lmax or Lo.

As in the other groups, marked PSS appeared during acute ischemia and it continued in five of the nine subjects at five minutes reperfusion. By 60 min reperfusion, seven of the nine animals had zero PSS and the median value was less than that which occurred during ischemia.

Ees did not change during ischemia.

After reperfusion, Ees was similar to control. At 60 $\mathrm{min}$. reperfusion, it was less than those values obtained during ischemia.

There were no changes in the non-ischemic (LX) segment.

\section{Intergroup comparison: (Tables $I I I, I V$ )}

The weight of the animals was similar in the different groups. Fewer animals required defibrillation after reperfusion was started in the halothane group (three of 10) than in the sevoflurane (eight of nine) and the control group (five of five). The halothane group also required less lidocaine for treatment of dysrhytthmias than the other two groups. The median amount of joules required for defibrillation was less in the halothane group than the control group but was no different from the sevoflurane group.

Intergroup comparison of regional myocardial function of the ischemic (LAD) segment was performed (dL, $\mathrm{dL} \%, \mathrm{RSW}, \mathrm{Lo}$ and PSS). At $\mathbf{5}$ and $\mathbf{3 0} \mathrm{min}$ after reperfusion was initiated, $\mathrm{dL} \%$ in the halothane group had recovered to median values of 88 and $92 \%$ of the initial (control) values respectively. These were greater than the 40 and $56 \%$ of the sevoflurane group, which in turn did not differ from the control group. Similarly, RSW in the halothane group recovered to 97,82 and $108 \%$ of the initial values by 5,30 and 60 min reperfusion. These values were different from those recorded in the sevoflurane group (36, 36 and $54 \%$ ) which again were not different from those recorded for the control group.

Post-systolic shortening during the ischemic phase was less in the sevoflurane group than in the control group (median values 44 vs $85 \%$ ).
Five minutes after reperfusion, Lo in the halothane group was less than that of the control group (105 vs $153 \%)$.

\section{Discussion}

Results from this study showed that neither halothane nor sevoflurane protected the myocardium against the effects of severe ischemia. Also, during reperfusion, sevoflurane was associated with more arrhythmias than was halothane and recovery of the stunned myocardium was less rapid in the presence of sevoflurane compared to halothane.

During acute myocardial ischemia, regional systolic function was reduced in the all three experimental groups and there was no difference in the extent of the regional dysfunction when the three groups were compared. The ischemic paralysis of the LAD segment was reflected by decreased regional stroke work and diastolic dysfunction occurred as shown by the increased PSS. The latter signifies shortening of the ischemic segment after aortic valve closure and therefore does not represent external cardiac work.

In all three groups the median Ees increased numerically during acute ischemia but this did not reach statistical significance. This apparent increase in end-systolic elastance is explained by the interaction between the ischemic paralysis of the LAD region and the increase in LV pressure during systole. Only a small amount (if any) regional shortening occurred during ischemia but the segment is subjected to the increased LV pressure. Because Ees is calculated as the quotient of LV pressure and regional shortening, the mathematical value increases but this is a passive interaction rather than an active process. This interpretation is supported by the decrease in regional SW, which occurred during acute ischemia.

Results recorded during acute severe ischemia in our experiments correspond with previously published studies. ${ }^{20,23}$ In the isolated rat heart we have also demonstrated that halothane offered no myocardial protection during acute ischemia. ${ }^{2}$ However, a more recent publication showed that sevoflurane lessens the degradation of high energy phosphates in the myocardium during three minutes of ischemia. ${ }^{24}$ The authors speculated that this could be explained by a reduction in myocardial contractility, reduced sympathetic stimulation, and increased vagal tone or by the effect of sevoflurane on coronary flow. However, their study used only a short period of ischemia and, furthermore, these beneficial effects were only shown at 2 MAC. In addition, they did not evaluate regional function during acute ischemia and it is therefore not known whether this conservation of energy substrate 
would have led to preservation of regional function. Yet another study suggested that halothane administered before hypoperfusion of guinea pig hearts resulted in decreased release of adenosine, inosine and lactate during ischemia and reperfusion. ${ }^{25}$ These authors came to the conclusion that the reduction in myocardial work associated with halothane, given before the onset of ischemia, could partially explain the improved function which was recorded once reperfusion was established in the presence of halothane. This differs from our results in the isolated rat heart model where we failed to show that halothane could preserve the high energy phosphate content during ischemia. ${ }^{2}$

During the reperfision section of the protocol, differences between halothane and sevoflurane emerged. The incidence of arrhythmias was less in the halothane experiments and, in addition, when arrhythmias did appear, they seemed to occur later during reperfusion when compared with the sevoflurane group. In the latter, the majority of animals had severe ventricular arrhythmias upon reperfusion and it is our impression (this was not specifically measured) that the arrhythmias occurred earlier during reperfusion. The beneficial effect of halothane on arrhythmias during reperfusion has previously been recorded in a similar model in which we demonstrated that as little as $0.2 \%$ halothane reduced the incidence of ventricular extra-systolic beats, ventricular tachycardia and fibrillation during reperfusion. ${ }^{4}$ A study in guinea pig hearts also demonstrated less arrhythmias if reoxygenation occurred in the presence of halothane than with isoflurane ${ }^{26}$ or in the absence of halothane. ${ }^{27}$

Reperfusion arrhythmias are preceded by an increase in intracellular calcium ${ }^{28-30}$ and drugs or interventions which control the calcium transients, can prevent or reduce the incidence of arrhythmias. ${ }^{31,32}$ The administration of halothane or isoflurane results in decreased intracellular calcium. ${ }^{18,33}$ but whether this can be explained by the effect of the inhalational anesthetics on the sarcolemma, ${ }^{13,14,15,33-40}$ sarcoplasmic reticulum ${ }^{41-47}$ or both, remains undecided.

No other study has examined the effect of sevoflurane on reperfusion arrhythmias. At best, comparison can be made with isoflurane, which, like sevoflurane, is an ether. A number of studies, in various models and with different aims, demonstrated that halothane is more potent in depressing intracellular calci$u^{8,26,27,37,44,47}$ and results from this study indirectly support this conclusion inasmuch as fewer arrhythmias occurred in the presence of halothane.

Evaluation of systolic regional myocardial function demonstrated that halothane was associated with a more rapid and complete recovery of function during reperfusion. Recovery in the halothane group did not differ from the control group although the median values for the control group resemble those of the sevoflurane animals. The small number of experiments , especially in the control group, and the scatter of the data could explain the absence of statistical significance in recovery patterns when halothane was compared with control.

Diastolic dysfunction, gauged by PSS, recovered equally well among the three experimental groups. This can be viewed as being at variance with the hypothesis that inhalation anesthesia limited the reperfusion calcium overload as there are data which suggest that diastolic dysfunction relates to relative cytosolic calcium overload. ${ }^{47,48}$ However, the index of diastolic dysfunction used in our experiments may not be sufficiently sensitive to register small differences if they were present.

Heart rates and arterial pressures differed between the two groups before ischemia and differences in regional myocardial oxygen consumption could explain the differences in regional function we observed during reperfusion. However, we used a 15 min normothermic total occlusion model which resulted in similar degrees of ischemia in the three groups. During ischemia little regional stroke work was performed and oxygen consumption would be negligible. It is, therefore, unlikely that differences in regional work (and myocardial oxygen consumption) which may have occurred before occlusion of the LAD artery or differences in oxygen consumption during ischemia could explain the differential results we recorded.

Three points of criticism can be leveled against this study. The control group was not part of the formal randomization and was performed $a d$ hoc between the randomized study. However, the model is well established, gives consistent results and data from this control group are similar to those previously recorded. ${ }^{4}$ Another limitation is that the inhalational drugs have different effects on the circulation and they both differ from the control group. Hence, the base-line values among the three groups are not necessarily similar. In addition, halothane could exert a myocardial depressant effect but at the same time protect against myocardial stunning. ${ }^{4}$ To overcome some of these limitations, the subsequent steps for each group were primarily compared to its own base-line value. For intergroup comparison, subsequent values were normalised for its own control values. Finally, our data do not allow us to address possible mechanisms. This was a comparative study and additional studies will have to be performed to elucidate the mechanisms involved. 
In this model of acute and severe myocardial ischemia and $60 \mathrm{~min}$ reperfusion, halothane was associated with fewer ventricular arrhythmias during reperfusion than was sevoflurane. Data further suggest that reperfusion systolic dysfunction recovered more rapidly in the presence of halothane than sevoflurane. However, extrapolation from this acute animal data to human patients with (chronic) ischemic heart disease must be done with the necessary caution.

\section{References}

1 Coetzee A, Skein W, Genade S, Lochner A. Enflurane and isoflurane reduce reperfusion dysfunction in the isolated rat heart. Anesth Analg 1993; 76: 602-8.

2 Coetzee A, Brits W, Genade S, Lochner A. Halothane does have protective properties in the isolated ischemic rat heart. Anesth Analg 1991; 73: 711-9.

3 Warltier DC, Al-Wathique MH, Kampine JP, Schemling $W T$. Recovery of contractile function of stunned myocardium in chronically instrumented dogs is enhanced by halothane or isoflurane. Anesthesiology $1988 ; 69: 552-5$.

4 Coetzee $A$, Moolman J. Halothane and the reperfusion injury in the intact animal model. Anesth Analg 1993; 76: 734-4.

5 Bolli R. Mechanisms of myocardial "stunning". Circulation 1990; 82: 723-8.

6 Bolli R, Patel BS, Jeroudi MO, Lai EK, McCay PB. Demonstration of free radical generation in "stunned" myocardium of intact dogs with use of spin trap -Phenyl $\mathrm{N}$-tert-butyl nitrone. J Clin Invest 1988; 82: 476-85.

7 Bolli $R, Z h u W-X$, Hartley CJ, et al. Attenuation of dysfunction in the postischemic "stunned" myocardium by dimethylthiourea. Circulation 1987; 76: 458-68.

8 Bolli $R$, Patel BS, Zhu W-X, et al. The iron chelator desferrioxamine attenuates postischemic ventricular dysfunction. Am J Physiol 1987; 253: H1372-80.

9 Przyklenk K, Kloner RA. Superoxide dismutase plus catalase improves contractile function in the canine model of "stunned myocardium". Circ Res 1986; 58: 148-56.

10 Kusuoka H, Porterfield JK, Weisman HF, Weisfeldt $M L$, Marban E. Pathophysiology and pathogenesis of stunned myocardium. Depressed $\mathrm{Ca}^{2+}$ activation of contraction as a consequence of reperfusion- induced cellular calcium overload in ferret hearts. J Clin Invest 1987; 79: 950-61.

11 Nayler WG, Panagiotopoulos $S$, Elz JS, Daly $M J$. Calcium-mediated damage during post-ischaemic reperfusion. J Moll Cell Cardiol 1988; 20(SupplII): 41-54.

12 Kukreja $R C$, Hess $M L$. The oxygen free radical system: from equations through membrane-protein interactions to cardiovascular injury and protection. Cardiovasc Res 1992; 26: 641-55.
13 Wilde $D W$, Knight $P R$, Sheth $N$, Williams $B A$. Halothane alters control of intracellular $\mathrm{Ca}^{2+}$ mobilization in single rat ventricular myocytes. Anesthesiology 1991; 75: 1075-86.

14 Ukemoto , Yatani A, Arimura H, Toshitake J. Reduction of the slow inward current of isolated rat ventricular cells by thiamylal and halothane. Acta Anesthesiol Scand 1985; 29: 583-6.

15 Bosnjak $Z K$, Kampine JP. Effects of halothane on transmembrane potentials, $\mathrm{Ca}^{2+}$ transients, and papillary muscle tension in the cat. Am J Physiol 1986; 251: H374-81.

16 Lynch C III, Vogel S, Sperelakis N. Halothane depression of myocardial slow action potentials.

Anesthesiology 1981; 55: 360-8.

17 Mongo $K G$, Vassort $G$ Inhibition by alcohols, halothane and chloroform of the $\mathrm{Ca}$ currents in single frog ventricular cells. J Moll Cell Cardiol 1990; 22: 939-53.

18 Bosnjak ZJ, Aggarwal A, Turner LA, Kampine JM, Kampine JP. Differential effects of halothane, enflurane and isoflurane on $\mathrm{Ca}^{2+}$ transients and papillary muscle tension in guinea pigs. Anesthesiology 1992; 76: 123-31.

19 Hoka S, Bosnjak ZJ, Kampine JP. Halothane inhibits calcium accumulation following myocardial ischemia and calcium paradox in guinea pig hearts. Anesthesiology 1987; 67: 197-202.

20 Hatakeyama N, Ito $\Upsilon$, Momose $\Upsilon$. Effects of sevoflurane, isoflurane and halothane on mechanical and electrophysiologic properties of canine myocardium. Anesth Analg 1993; 76: 1327-32.

21 Hatakeyama $N$, Momose $Y$, Ito $\Upsilon$. Effects of sevoflurane on contractile responses and electrophysiologic properties in canine single cardiac myocytes. Anesthesiology $1995 ; 82: 559-65$.

22 Glower DD, Spratt JA, Kabas JS, Davis JW, Rankin JS. Quantification of regional myocardial dysfunction after acute ischemic injury. Am J Physiol 1988; 255: H85-93.

23 Osakada G, Hess OM, Gallagher KP, Kemper WS, Ross $J$ $J r$ End-systolic dimension-wall thickness relations during myocardial ischemia in conscious dogs. A new approach for defining regional function. Am J Cardiol $1983 ; 51: 1750-8$.

24 Takabata $O$, Ichihara $K$, Ogawa $H$. Effects of sevoflurane on ischaemic myocardium in dogs. Acta Anesthesiol Scand 1995; 39: 449-6.

25 Buljubasic N, Stowe DF, Marijic J, Roerig DL, Kampine JP, Bosnjak ZJ. Halothane reduces release of adenosine, inosine, and lactate with ischemia and reperfusion in isolated hearts. Anesth Analg 1993; 76: 54-62.

26 Marijic J, Stowe DF, Turner LA, Kampine JP, Bosnjak ZJ. Differential protective effects of halothane and isoflurane against hypoxic and reoxygenation injury in the isolated guinea pig heart. Anesthesiology 1990; 73: 976-83. 
27 Buljubasic N, Marijic J, Stowe DF, Kampine JP, Bosnjak $Z J$. Halothane reduces dysrhythmias and improves contractile function after global hypoperfusion in isolated hearts. Anesth Analg 1992; 74: 384-94.

28 Brooks WW, Conrad CH, Morgan JP. Reperfusion induced arrhythmias following ischemia in intact rat heart: role of inracellular calcium. Cardiovasc Res 1995; 29: 536-42.

29 Kibara $\Upsilon$, Morgan JP. Intracellular calcium and ventricular fibrillation. Studies in the aqueorin-loaded isovolemic ferret heart. Circ Res 1991; 68: 1378-89.

30 Kihara $\Upsilon$, Grossman W, Morgan JP. Direct measurement of changes in intracellular calcium transients during hypoxia, ischemia and reperfusion of the intact mammalian heart. Circ Res 1989; 65: 1029-44.

31 Brooks WW, Verrier $R L$, Lown B. Protective effect of verapamil on vulnerability to ventricular fibrillation during myoardial ischemia and reperfusion. Cardiovasc Res 1980; 14: 295-302.

32 Tosaki A, Hearse DJ. Protective effect of transient calcium reduction against reperfusion-induced arrhythmias in rat hearts. Am J Physiol 1987; 253: H225-33.

33 Housmans PR. Negative inotropy of halogenated anesthetics in ferret ventricular myocardium. Am J Physiol 1990; 259: H827-34.

34 DeTraglia $M C$, Komai $H$, Redon D, Rusy BF. Isoflurane and halothane inhibit tetanic contractions in the rabbit myocardium in vitro. Anesthesiology 1989; 70: $837-42$.

35 Drenger $B$, Quigg $M$, Blanck TJJ. Volatile anesthetics depress calcium channel blockers binding to bovine cardiac sarcolemma. Anesthesiology 1991; 74: 155-65.

36 Hoebner PJ, Quigg M, Blanck TJJ. Halothane depresses D600 binding to bovine heart sarcolemma. Anesthesiology 1991; 75: 1019-24.

37 Komai $H, R$ usy BF. Negative inotropic effects of isoflurane and halothane in rabbit papillary muscles. Anesth Analg 1987; 66: 29-33.

38 Terrar DA, Victory JGG. Effects of halothane on membrane currents associated with contraction in single myocytes isolated from guinea-pig ventricle. $\mathrm{Br}$ J Pharmacol 1988; 94: 500-8.

39 Baum VC. Will the calcium channel agonist BAY K8644 inhibit halothane-induced impairment of calcuim current? Anesth Analg 1992; 74: 865-9.

40 Eskinder H, Rusch NJ, Supan FD, Kampine JP, Bosnjak $Z$. The effects of volatile anesthetic on $\mathrm{L}$ - and T-type calcium channel currents in canine cardiac Purkinje cells. Anesthesiology 1991; 74: 919-26.

41 Blanck TJ, Petersen CV, Baroody B, Tegazzin V. Halothane, enflurane, and isoflurane stimulate calcium leakage from rabbit sarcoplasmic reticulum. Anesthesiology 1992; 76: 813-21.
42 Casella ES, Suite NDA, Fisher YI, Blanck TJJ. The effect of volatile anesthetics on the $\mathrm{pH}$ dependance of calcium uptake by cardiac sorcoplasmic reticulum. Anesthesiology 1987; 67: 386-90.

43 Herland JS, Julian FJ, Stephenson DG. Halothane increases $\mathrm{Ca}^{2+}$ efflux via $\mathrm{Ca}^{2+}$ channels of sarcoplasmic reticulum in chemically skinned rat myocardium. J Physiol 1990; 426: 1-18.

44 Housmans PR, Murat I. Comparative effects of halothane, enflurane, and isoflurane at equipotent anesthetic concentrations on isolated ventricular myocardium of the ferret. II. Relaxation. Anesthesiology 1988; 69: 464-71.

45 Katsuoaka $M$, Obnishi ST. Inhalation anesthetics decrease calcium content of cardiac sarcoplasmic reticulum. Br J Anaesth 1989; 62: 669-73.

46 Krane EJ, Su JY. Comparison of the effects of halothane on skinned myocardial fibers from newborn and adult rabbit. II. Effects on sarcoplasmic reticulum. Anesthesiology 1989; 71: 103-9.

47 Komai $H, R$ usy BF Direct effect of halothane and isoflurane on the function of the sarcoplasmic reticulum in intact rabbit atria. Anesthesiology 1990; 72: 694-98.

48 Bourdillon PD, Poole-Wilson PA The effects of verapamil , quiescence and cardioplegia on calcium exchange and mechanical function in ischemic rabbit myocardium. Circ Res 1982; 50: 360-68.

49 Shen $A C$, Jennings $R B$ Kinetics of calcium accumulation in acute myocardial ischemic injury. Am J Pathol 1972; 67: 441-52. 